\title{
TV/Series
}

1 | 2012

Les Séries télévisées américaines contemporaines :

entre la fiction, les faits, et le réel

\section{« Mars women » et « gossip girls» : les ambivalences des héroïnes de séries adolescentes contemporaines}

Pierre-Olivier Toulza

\section{CpenEdition}

\section{Journals}

Édition électronique

URL : http://journals.openedition.org/tvseries/1213

DOI : $10.4000 /$ tvseries.1213

ISSN : 2266-0909

Éditeur

GRIC - Groupe de recherche Identités et Cultures

\section{Référence électronique}

Pierre-Olivier Toulza, « « Mars women » et « gossip girls » : les ambivalences des héroïnes de séries adolescentes contemporaines », TV/Series [En ligne], 1 | 2012, mis en ligne le 15 mai 2012, consulté le 19 avril 2019. URL : http://journals.openedition.org/tvseries/1213; DOI : 10.4000/tvseries.1213

\section{(c) $($ ) $(3)$}

TV/Series est mis à disposition selon les termes de la licence Creative Commons Attribution - Pas d'Utilisation Commerciale - Pas de Modification 4.0 International. 


\section{«Mars women » et « gossip girls 》: les ambivalences des héroïnes de séries adolescentes contemporaines}

Pierre-Olivier ToulzA

Le genre de la série adolescente, qui a aujourd'hui atteint une forme de stabilité, comble un manque en matière de représentation d'enjeux identitaires qui sont rarement abordés avec une telle complexité par les fictions sérielles contemporaines. La formule générique du teen drama s'inspire beaucoup de celle du soap opera, tout en opérant de fréquentes alliances avec le fantastique et la science fiction. Le public visé par ces séries comprend les adolescents, mais aussi les enfants et les jeunes adultes, et plusieurs stratégies narratives peuvent être repérées dans ces séries pour attirer l'attention de ces spectateurs spécifiques. En outre, parce que ces séries tentent de séduire un public de jeunes filles et de jeunes femmes, elles mettent en scène des personnages féminins forts, dans la lignée des séries de la vague girlie des années 1990. Toutefois, à la différence des fictions des décennies antérieures comme Buffy contre les vampires, par exemple, les séries adolescentes contemporaines mettent en scène de façon récurrente l'intersection des problématiques de genre, d'ethnicité, de classe sociale et de sexualité.

es adolescents ordinaires qui, à l'aide d'une intelligence hors du

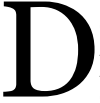
commun ou des pouvoirs surnaturels qui sont les leurs, parviennent à résoudre des enquêtes que les meilleurs détectives sont incapables de démêler ou à sortir indemnes d'attaques de vampires, de morsures de loups-garous et autres situations fort périlleuses : ce type de péripétie court de Buffy contre les vampires (WB puis UPN, 1997-2003) à The Vampire Diaries (CW, 2009-) en passant par Veronica Mars (UPN puis CW, 2004-2007). Saturées d'intrigues rocambolesques, peuplées de jeunes gens échangeant des répliques brillantes et pleines d'humour qui, dans le monde réel, ne seraient guère de leur âge, les séries adolescentes américaines, parfois vantées pour la qualité de leur écriture et la

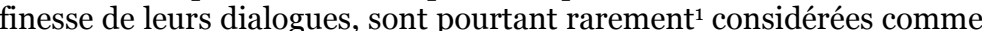
des fictions télévisuelles aussi riches que les séries dramatiques comme Six Feet under (HBO, 2001-2007), Mad Men (AMC, 2007-) ou Les Soprano (HBO, 1999-2007). Parce que ces dramas sont largement consacrés à la caractérisation psychologique des personnages, ils nous offrent des fictions plantureuses qui paraissent refléter avec précision le monde actuel, tandis que les séries adolescentes épouseraient les contours d'un genre mineur, fondé sur un divorce entre l'univers

${ }^{1}$ A l'exception de Buffy contre les vampires, qui a suscité un grand nombre de publications universitaires aux Etats-Unis. 
fictionnel et le monde actuel, et partant sur la référence à des mondes de fantaisie.

Pourtant, loin d'être destinée aux seuls adolescents, la teen series est un genre visant un vaste public, essentiellement - mais pas uniquement - féminin et qui, dans le meilleur des cas, place au centre de son univers des jeunes femmes « confrontées à des problèmes émotionnels, sociaux et psychologiques qui sont spécifiquement en rapport avec le fait qu'elles soient des femmes ${ }^{2}$ », problèmes qu'elles tentent parfois de résoudre, mais généralement en vain. Un bon nombre de séries dramatiques contemporaines - Dexter (Showtime, 2006-), Breaking Bad (AMC, 2008-), 24 heures chrono (Fox, 20012010), etc. - invitent spectatrices et spectateurs à compatir aux doutes et dilemmes qui sont ceux d'hommes blancs, hétérosexuels et pères de famille. A l'inverse, les séries adolescentes des années 2000, au plus près de l'expérience des femmes - et aussi des hommes d'aujourd'hui, explorent systématiquement l'intersection entre les identités sexuées, les identités ethniques, et les identités de classe. Si ces programmes suscitent tant d'intérêt sur les forums du web ou dans les journaux populaires, par exemple, c'est justement parce qu'ils comblent un manque en matière de représentation d'enjeux identitaires rarement abordés avec une telle complexité par les fictions sérielles d'aujourd'hui.

\section{Un genre protéiforme}

La bibliographie française sur les séries adolescentes américaines est inexistante. On peut se référer à quelques titres américains $^{3}$, mais qui abordent rarement la teen series comme un genre. De très nombreuses publications existent sur la série Buffy contre les vampires, mais peu d'articles et d'ouvrages prennent en compte l'histoire du genre, et tentent d'établir sa formule sémanticosyntaxique. Pourtant, si la teen series n'est pas un genre très ancien, il semble que sa formule est aujourd'hui parvenue à une sorte de stabilité.

Prenant acte que le public adolescent est devenu de plus en plus important pour le cinéma depuis les années 1950, les fictions destinées à des adolescents ont colonisé les grilles de programmation des télévisions à partir des années 1960. Aux Etats-Unis, la série The

\footnotetext{
${ }^{2}$ Nous paraphrasons la définition que propose Jeanine Basinger du woman's film. Jeanine Basinger, A Woman's View: How Hollywood Spoke to Women, 1930-1960, Londres, Chatto and Windus, 1993, p. 20.

3 On peut notamment se reporter aux publications suivantes: Rachel Moseley, « The Teen Series », in Glen Creeber (dir.), The Television Genre Book, Londres, BFI, 2001, p. 41-43; Glyn Davis and Kay Dickinson (dir), Teen TV - Genre, Consumption and Identity, Londres, BFI, 2004; Mary Celeste Kearney, "the Changing Face of Teen Television, or Why We All Love Buffy ", in Elana Levine et Lisa Parks (dir.), Undead TV - Essays on 'Buffy The Vampire Slayer', Durham et Londres, Duke University Press, 2007, p. 17-41.
} 
Monkees (NBC, 1966-1968) fait figure de précurseur. Dans un format de 30 minutes, cette série inspirée par le film A Hard Day's Night (Richard Lester, 1964) s'intéresse à un groupe de jeunes musiciens et mêle aventures comiques et performances musicales. Deux genres distincts prennent leur essor dès cette époque, que l'on peut distinguer par le ton et le format. Les sitcoms, particulièrement ceux centrés sur les relations familiales, ont souvent mis en scène des personnages d'adolescents, à l'instar de The Brady Bunch (ABC, 1969-1974) et The Partridge Family (ABC, 1970-1974). A partir des années 1980 se développent des sitcoms s'intéressant spécifiquement aux vies et aux amours d'adolescents, qui ciblent un public jeune, à l'instar de Sauvés par le gong (NBC, 1989-1993), qui accompagnait quelques adolescents types, repris au répertoire des teen movies (le bon élève, la cheer leader, la peste, etc.), depuis leur années high school et jusqu'à l'université.

Par ailleurs, au fil des décennies, les soap operas ont accru au sein de leurs intrigues la présence et le rôle des personnages adolescents, au point que certains programmes comme Beverly Hills 90210 (Fox, 1990-2000) et Melrose Place (Fox, 1992-1999) puissent à leur tour traiter, à partir de ces personnages spécifiques, de problèmes émotionnels, affectifs et sociaux complexes (grossesse d'adolescentes, addiction à la drogue, adoption, divorce, suicide, etc.) empruntés au répertoire du soap opera. Grâce à ces précurseurs, le teen drama se développe de manière exponentielle dans les années 1990 et $2000^{4}$. Les séries qui ne témoignent pas d'une alliance avec un autre genre sont, dans leur formule narrative, très proches du soap opera: si parfois une intrigue est résolue dans un épisode de 42 minutes, l'accent porté sur la répétition et le retard de toute résolution les rapprochent de l'absence de clôture qui définit la spécificité du soap opera. Comme dans le soap, les relations sentimentales et affectives donnent lieu à une intense activité narrative, et on peut prendre pour règle qu'une teen series explorera toutes les possibilités combinatoires en ce domaine: dans Secret Life of the American Teenager (ABC Family, 2008-), par exemple, Ricky, le père biologique du bébé d'Amy, l'héroïne, entretient une relation avec la jeune Adrian. Mais cela ne l'empêche guère de courtiser Grace, et même de séduire Lauren, la meilleure amie d'Amy. Comme dans le soap, ensuite, le dialogue prime sur l'action, au point même que les conversations puissent tenir lieu d'action. On ne saurait prétendre qu'il ne se passe rien dans les teen series, bien au contraire, et on retrouve dans ces feuilletons les situations rocambolesques propres au soap, mais qui deviennent surtout des prétextes à des commentaires sans fin : toutes les héroïnes de teen series sont des sortes de gossip girls. Des séries comme Secret

4 Pour une liste des principaux teen dramas américains d'aujourd'hui, voir par exemple http://en.wikipedia.org/wiki/List_of_teen_dramas. Page consultée le o1 juin 2011. 
Life et Gossip Girl (CW, 2007-) méconnaissent généralement la combinaison de logique sérielle et feuilletonnante qui est le propre des néo-séries américaines tant discutées aujourd'hui : dans le cadre d'un épisode, il arrive souvent qu'aucune ligne narrative ne trouve de résolution, et les intrigues pourraient être, littéralement, sans fin. Les différences (d'ordre industriel et narratif) entre soap opera et teen series sont bien entendu légion : les horaires de diffusion de ces deux genres ne sont pas identiques; les teen series connaissent une fin, à l'inverse du soap ; elles ne sont pas destinées uniquement aux femmes. En proposant cette analogie entre teen series et soap, il ne s'agit nullement faire de la série adolescente contemporaine un dinosaure de la sérialité télévisuelle. L'adaptation du modèle soap operatique me semble au contraire une solution inventive élaborée depuis une quinzaine d'années par l'industrie de la télévision américaine pour s'adresser à un public spécifique, les jeunes.

L'influence du soap opera, pour être déterminante, n'est pas la seule à s'exercer sur les séries adolescentes contemporaines : il ne faut pas oublier que, dès les années 1990, la teen series s'allie fréquemment à d'autres genres sériels, principalement le fantastique et la science fiction, dans Buffy, Angel (WB, 1999-2004) ou encore Charmed (WB, 1998-2006). L'explication la plus convaincante de ces alliances a été donnée par Rachel Moseley, pour qui

[...] la plupart des séries adolescentes traitent de questions de différence, d'altérité, de pouvoir accru, et l'impact de ces trois facteurs sur les relations entre individus : c'est pourquoi un nombre significatif de ces programmes se fondent sur d'autres genres télévisuels, en utilisant la puissance surnaturelle comme un motif permettant d'explorer ces problématiques. De nombreuses séries donnent ainsi l'impression qu'être adolescent c'est presque ne pas être humain5.

\section{Cibler les jeunes adultes}

La forme des séries adolescentes semble ainsi largement déterminée par l'âge des spectateurs, mais l'étude des stratégies des chaînes et des annonceurs publicitaires montre que l'étiquette de teen series ne reflète qu'imparfaitement le public ciblé. A la fin des années 1990, le fort développement des séries adolescentes répond à une stratégie industrielle complexe : en effet, les adolescents américains regardent moins la télévision que d'autres tranches d'âge, et le grand nombre de séries centrées sur des adolescents n'a pas permis d'inverser cette tendance. D'autres loisirs (notamment internet) sont privilégiés par ces jeunes spectateurs. En raison aussi du nombre important de teen series disponibles à la même heure sur les différentes chaînes, aucune teen series ne pourrait exister en ciblant uniquement un public

5 Rachel Moseley, « The Teen Series », op. cit., p. 43. 
adolescent. De même, les annonceurs publicitaires - parties prenantes, avec les chaînes et les sociétés de production, du processus industriel de création d'une série ${ }^{6}$ - revoient depuis plusieurs années leurs modes d'annonce en direction des adolescents, et se détournent de la télévision traditionnelle pour concentrer leurs efforts sur les nouveaux médias. A cette époque, les chaînes et les annonceurs publicitaires se détournent d'une stratégie de simple segmentation des publics, qui consiste à viser une cible spectatorielle, même étroite, définie en termes d'âge, de sexe, et d'origine ethnique, et surtout de pouvoir d'achat des spectateurs. Ils privilégient plutôt la création d'une coalition audience : il s'agit alors, comme l'a montré Jim Collins ${ }^{7}$, de reconfigurer la politique de segmentation des publics de façon à obtenir des résultats voisins de ceux générés antérieurement par la politique de mass audience ${ }^{8}$. Mais le but est désormais de fédérer plusieurs groupes démographiques susceptibles d'être intéressés par des programmes identiques. La chaîne WB, un grand diffuseur de séries adolescentes dans les années 1990, a ainsi tenté de fidéliser avec ses programmes un public de pré-adolescents, d'adolescents et de jeunes adultes, c'est-à-dire des individus qui ne sont pas reliés par l'âge, mais par une même sensibilité jeune. Pour plusieurs raisons, liées notamment à des évolutions de la société, le cœur de cible des séries de ce type de chaîne est aujourd'hui constitué par les enfants de 9 à 15 ans. D'autre part, si l'adolescence est une catégorie qui englobe désormais les enfants à partir de 9 ans, il faut noter que, à l'autre bout du spectre, les chaînes ciblent désormais les adultes qui retardent l'entrée dans l'âge adulte, et des groupes qui rejettent les normes et les formes traditionnelles du statut d'adulte défini par le patriarcat, notamment les féministes et des catégories socialement opprimées comme les lesbiennes et les homosexuels. Mais ce sont aussi, plus largement, tous les adultes qui adhèrent aux normes patriarcales qui sont encouragés à adopter un mode de vie et une sensibilité jeune, notamment dans leur style de consommation 9 .

Plusieurs stratégies, dans les teen series contemporaines, sont utilisées pour susciter l'intérêt d'adultes rejetant l'idéologie majoritaire : tout d'abord, le faible nombre dans les teen series de familles traditionnelles, par rapport aux séries dramatiques classiques.

${ }^{6}$ Sur ce point, cf. Eric Vérat, « États-Unis, le règne des saisons et la galaxie des auteurs », in MédiaMorphoses, Hors-Série n.3, « Les raisons d'aimer les séries télé », 2007.

7 Jim Collins, "Television and postmodernism », in Robert C. Allen, Channels of Discourse, Reassembled - Television and Contemporary Criticism, Londres, Routledge, 1992, p. 327353.

8 Avant le développement du câble et l'apparition de nouveaux réseaux, les networks historiques, jusqu'aux années 1980, tentaient de viser le public le plus large possible, indépendamment de critères démographiques.

9 Nous résumons dans ce paragraphe la thèse exposée par Mary Celeste Kearney dans « The Changing Face of Teen television, or Why We All Love Buffy ", in Elana Levine et Lisa Parks, op. cit., p. 17-41. 
Veronica Mars, dans la série éponyme, habite avec son seul père, et tous les héros et héroïnes de Gossip Girl évoluent dans des familles monoparentales. L'écart entre les générations est souvent brouillé dans la fiction : dans la série Gilmore Girls (WB puis CW, 2000-2007), les girls du titre sont une mère et sa fille, qui portent le même prénom, et dans le pilote de la série, des hommes ne cessent de s'étonner que Lorelei puisse avoir une fille de quinze ans. Dans The Vampire Diaries, à la suite du décès de ses parents, la jeune Elena est élevée par sa tante d'une vingtaine d'années. D'autre part, et c'est une différence majeure avec le modèle soap opératique, l'homosexualité est souvent - quoique timidement - évoquée. Mais si l'homosexualité masculine est fréquente chez les adultes de teen series, l'homosexualité féminine ne franchit jamais le seuil des amours adolescentes. Dans The Vampire Diaries, le père de l'une des héroïnes a quitté sa femme pour un homme, tout comme le père de Blair, l'héroïne de Gossip Girl, parti vivre dans l'hexagone avec un mannequin français.

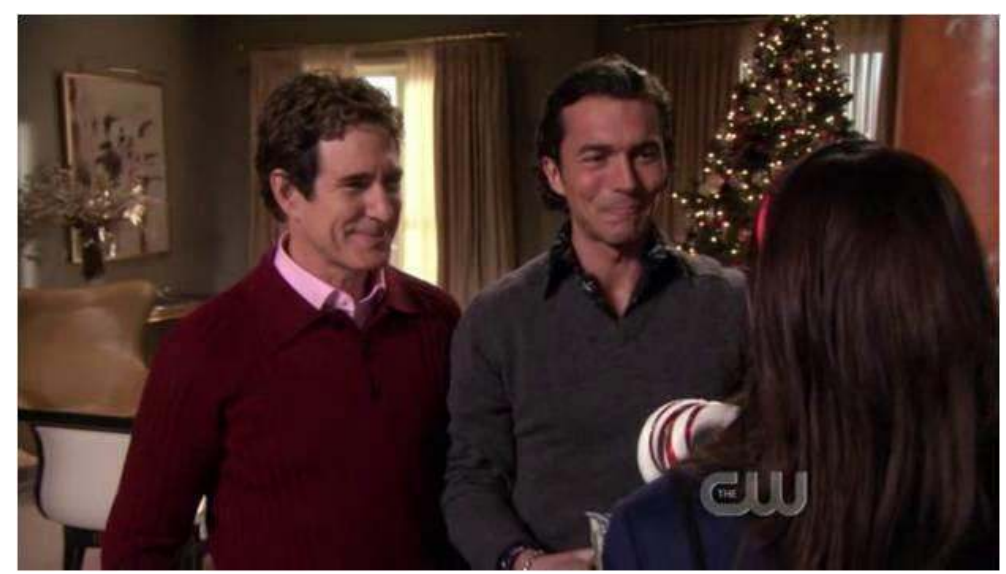

Gossip Girl

Dans un épisode de Veronica Mars (1.4), l'héroïne aide un camarade de classe à retrouver son père, devenu transsexuel, qui tente, de manière presque impossible, de concilier son identité de genre avec sa paternité. Le pilote de Glee (Fox, 2009-) nous présente de manière comique Rachel Berry, une jeune fille élevée par un couple d'hommes. Enfin, dans les teen series, on note fréquemment la constitution, à l'écart de la cellule familiale, de petites communautés soudées par un secret commun : dans The Vampire Diaries, la connaissance partagée par Elena et Bonnie de la présence de vampires dans leur petite ville scelle l'union des deux jeunes femmes dans une forme de sororité, de confiance et d'entraide, en dehors du cercle familial. Dans la seconde 
saison de cette série, le rôle des adultes est réduit à la portion congrue, et tous les enjeux importants sont traités entre adolescents. Immature et frivole dans les premiers épisodes de la série, Caroline devient ainsi une femme forte dans la seconde saison, prête à prendre, de son propre chef et à l'insu de tous, des risques importants pour épauler son ami Tyler lors d'une épreuve redoutable, sa première transformation en loup-garou (épisode 2.11).

\section{Une nouvelle vague girlie}

L'objectif à long terme des chaînes est donc de créer une coalition audience allant de 9 à 34 ans, en ciblant d'abord les préadolescents, puis en faisant en sorte que les teens et les jeunes adultes suivent. Enfin, et surtout, les chaînes dirigent tous leurs efforts vers les filles et les jeunes femmes. En se fondant sur des études sociologiques montrant que les jeunes filles achètent plus fréquemment, adoptent des habitudes de consommation plus jeunes, dépensent une plus large part de l'argent de leurs parents, restent davantage à la maison que les garçons, et passent plus de temps devant la télévision, la chaîne WB a tenté de cibler, dans les trois segments composant son public naturel, le public féminin ${ }^{10}$. WB put alors profiter de l'expérience de la Fox, qui dans les années 1990, avec Beverly Hills 90210, sut retenir l'attention des jeunes filles et des jeunes femmes, avec une série présentant des personnages féminins forts, et en portant l'accent sur l'amitié entre filles. A la suite de ce succès, dès le milieu des années 1990, les teen series commencèrent à mettre systématiquement sur le devant de la scène des personnages féminins complexes, et à fonder leurs intrigues sur les problèmes sentimentaux et émotionnels d'une ou de plusieurs héroïnes. Au début des années 1990, WB a été confortée dans cette stratégie par l'importance accrue accordée aux filles dans les pratiques culturelles de l'époque. Finalement, à partir de la série Buffy contre les vampires, la chaîne a obtenu des scores d'audience chez les adolescentes et les jeunes femmes supérieurs à ceux enregistrés auprès des garçons et des jeunes hommes. La série de Joss Whedon a donné lieu à un nombre important d'analyses aux Etats-Unis, qui ont souvent montré qu'elle mettait sur le devant de la scène une héroïne ambivalente, à la fois girlie girl et femme d'action ${ }^{11}$. Qu'en est-il des héroïnes des teen series contemporaines, quelques années après l'époque du girl power et des riot grrrls de la décennie 1990 ?

Un constat s'impose: la plupart des teen series mettent logiquement l'accent sur les personnages féminins plutôt que sur les garçons, et placent au centre de leur univers une femme confrontée à

${ }^{10} \mathrm{Cf}$. Mary Celeste Kearney, op. cit., p. 17-41.

${ }^{11}$ Cf. par exemple Elana Levine, "Buffy and the "New Girl Order": Defining Feminism and Femininity », in Elana levine et Lisa Parks, op. cit., p. 168-190. 
des problèmes spécifiquement féminins, mais imposent toutefois un cadrage violent qui relève autant d'une réaction à la vague girlie des années 1990 que de la tentative de séduire autant les femmes (le personnage principal est féminin) qu'un vaste public, indépendamment du sexe. La série Secret Life of the American Teenager est exemplaire de ces fictions globalement réactionnaires. L'héroïne, Amy, est une jeune lycéenne de 15 ans tombée enceinte à la suite d'une première relation sexuelle si furtive que la jeune fille n'est pas certaine qu'elle ait réellement eu lieu. Amy est confrontée à des problèmes qu'elle n'essaie jamais de résoudre: elle ne fait preuve d'aucune initiative, ne prend jamais de décision autonome. La série utilise la grossesse d'une adolescente pour traiter de façon normative de l'unique question qui, selon ce programme ultra-conservateur, devrait se poser aux adolescentes et aux jeunes femmes : la sexualité avant le mariage, qui nourrit la plupart des conversations. Le problème est vite résolu, puisque une relation avant le mariage définit nécessairement une jeune femme comme étant une traînée. Durant quelques épisodes est ainsi envisagée l'éventualité qu'Amy puisse rejoindre un lycée à horaires adaptés pour "jeunes femmes indépendantes ", mais son père et son futur mari la convainquent tous deux de ne pas aller dans cette école que les uns et les autres appellent slut school. Quand elle avoue finalement sa grossesse à ses parents (au bout du sixième épisode!), les options qui s'offrent à Amy sont réduites: l'avortement est une solution effleurée mais finalement rejetée par toutes et tous, et l'adoption de l'enfant à naître est tout autant dévalorisée. Un ami de lycée s'ouvre contre toute attente de son amour à la jeune fille : les uns et les autres pèsent alors le pour et le contre d'un mariage précoce de ces deux adolescents de quinze ans, qui permettrait à Amy de passer directement de l'autorité de son père à celle d'un mari. 


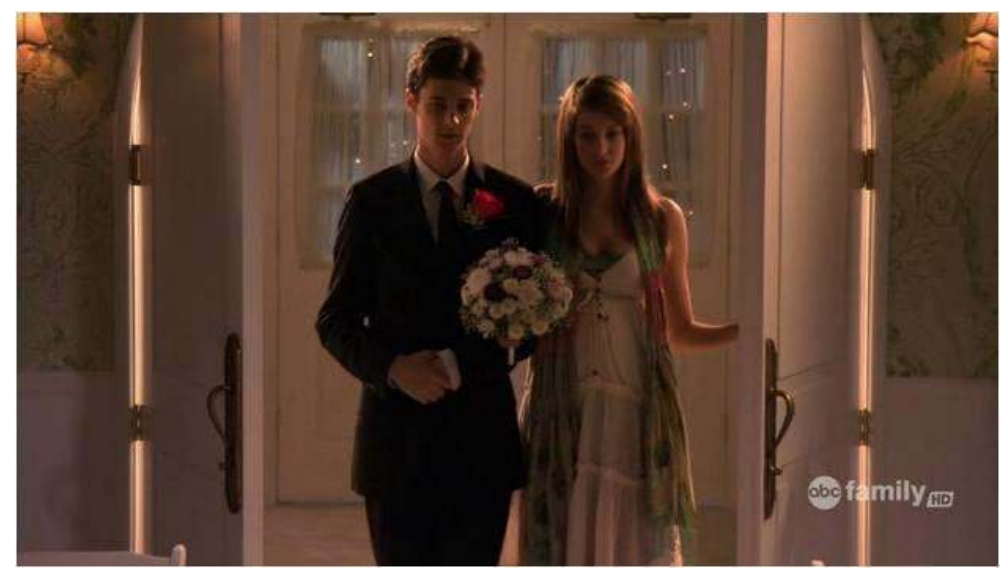

La séquence du mariage secret des deux adolescents (1.12) est représentative du refus d'adopter un point de vue féminin. L'homme accomplit toutes les actions (il paie le pasteur qui a accepté d'unir les adolescents ; il tend une bague à sa promise), ce qui est aisément concevable dans une série où l'un des pères se définit, avec l'approbation de son épouse, comme étant le roi de son foyer (le père de Grace, dans l'épisode 1.3). La jeune femme ne se marie pas car tel est son désir, mais parce que c'est la chose à faire pour sa famille, explique-t-elle au début de la scène. On peut être intrigué, dans cette séquence de mariage, par l'oubli d'Amy, qui n'a pas songé à apporter une bague pour son futur époux. Or la bague est un objet récurrent dans l'univers fictionnel de Secret Life : bague de pureté qu'accepte de porter l'une des héroïnes au début de la série, anneau de fiançailles, et enfin bague de mariage. L'oubli d'Amy est ainsi presque nécessaire, puisque l'objet signe la prise de possession symbolique de la jeune fille, qui ne saurait en toute logique passer une bague au doigt de son époux. Enfin, cette séquence est représentative de l'instrumentalisation des minorités dans les teen series conservatrices : homosexuels (le pasteur fait une plaisanterie sur la "Don't Ask Don't Tell Policy » de l'armée américaine), noirs, handicapés et aussi personnages au physique peu avantageux, qui assistent au mariage et offrent un contrepoint comique.

\section{Ambivalences}

Du fait de la cible très large qui est celle des teen series, une tension existe donc, au sein du genre, entre d'un côté des séries réactionnaires comme Secret Life of The American Teenager, et de l'autre une majorité de séries dans lesquelles les héroïnes sont loin 
d'être cantonnées à une telle passivité, ce qui n'empêche pas certaines contradictions. Le début du pilote de Veronica Mars est représentatif de certains de ces enjeux, et aussi de la différence entre les teen series contemporaines et les séries antérieures de la vague girlie, comme Buffy. Dans les premières minutes de cet épisode ${ }^{12}$, Veronica Mars se rend dans son lycée où elle découvre un garçon nu, ligoté comme un pirate au mât du lycée. La caractérisation de l'héroïne affiche d'emblée une certaine ambivalence. Physiquement, elle est une frêle jeune fille blonde, comme Buffy, qui semble tout naturellement adopter un comportement d'ordinaire réservé aux hommes. En arrivant en voiture, sa trajectoire est opposée à celle d'un groupe de cheer leaders sans cervelle ; elle est immédiatement dans l'action et la prise d'initiative, tandis que l'une de ses camarades de lycée se contente de demander pourquoi personne ne vient détacher l'adolescent; elle défie l'air de rien un jeune homme par la parole (un simple "move » suffit) et le geste (elle brandit une arme blanche). Ce début est ainsi caractéristique du contexte post-féministe dans lequel sont créées ces teen series : c'est sans effort particulier que Veronica s'approprie un rôle masculin (elle se définira, dans une conversation avec son père, comme une guerrière, une Mars woman) et témoigne d'un empowerment féminin. La série fait ainsi preuve d'une forme de déni des ambitions féministes des générations précédentes, puisque cela semble être du ressort de chaque femme de décider (c'est le cas de Veronica), ou non (comme la plupart des jeunes femmes de la série), d'adopter sans effort particulier un comportement jusqu'alors réservé aux hommes. L'empowerment ne passe pas par une résistance et un combat collectifs, qui semblent dépassés, puisqu'une jeune femme ordinaire placée dans une situation extraordinaire peut devenir forte par ses propres moyens. Dans Gossip Girl, également, le féminisme des générations passées est explicitement présenté comme un combat d'arrière-garde, dangereux même, lorsque l'un des personnages maléfiques de la série, la grandmère de l'héroïne, explique à sa petite-fille, pour légitimer ses actions scandaleuses : « une femme doit gagner le droit de créer ses propres règles ${ }^{13}$ ! »

Même dans une série progressiste comme Veronica Mars, une forme d'incohérence, probablement liée aux cibles spectatorielles visées par la série, se fait jour. Par opposition aux cheer leaders court vêtues, la tenue plutôt neutre de Veronica ne vient pas remettre en question son action par le rappel d'une féminité excessive, comme c'était le cas dans des séries antérieures, comme Wonder Woman (ABC puis CBS, 1975-1979) ou Drôles de dames (ABC, 1976-1981), où ce rappel visait à neutraliser le pouvoir subversif des héroïnes.

${ }^{12}$ Le début de l'épisode est visible sur le site Dailymotion :

http://www.dailymotion.com/video/x2ghku_vero-m-so1-eo1-1-3_shortfilms

${ }^{13}$ a woman has to earn the right to create her own rules ! (1.10) 
L'ambivalence de la série Buffy contre les vampires - une série pourtant louée par nombre de critiques féministes - a souvent été relevée : l'héroïne est tiraillée entre sa mission masculine (combattre des vampires et démons de toutes sortes) et des intérêts conventionnellement girly (les garçons, les vêtements, les cheer leaders). Veronica Mars, à l'inverse, semble considérer comme globalement atteints les objectifs de la seconde vague féministe, et ne joue pas de manière analogue sur la contradiction entre la mission de Veronica (résoudre des enquêtes fort compliquées) et son identité sexuée (c'est uniquement lors de flashbacks relatant un passé heureux qu'elle est présentée comme une jeune fille insouciante, préoccupée par les fêtes, les vêtements, et son boyfriend). Pourtant - et c'est bien là que réside une forme d'incohérence - dans plusieurs enquêtes, Veronica utilise son charme et un physique avantageux pour mener à bien sa mission. L'ambivalence est également sensible dans ses relations avec son père : le père de Veronica est un mari abandonné, un policier contraint à la démission, et dont les compétences professionnelles indubitables sont cependant complétées par l'aide indispensable de sa fille. Cette victimisation du personnage du père permet d'émouvoir le spectateur sur le sort de ce patriarche victime de ses bonnes intentions, et qui profite de l'abandon de son épouse pour nouer une relation bien étroite et quasi-incestueuse avec sa fille. Cet élément sémantique réactionnaire (le père, abandonné par une épouse que l'on croit ingrate, fait quasiment couple avec sa fille adolescente qui finit par prouver son innocence) est repris à la fin de la première saison de la série, quand le père de Veronica sauve in extremis l'héroïne d'une mort certaine (1.22).

En définitive, dans ces séries essentiellement destinées à un public féminin, l'analyse gender que fait Tania Modleski du soap opera est globalement pertinente ${ }^{14}$. L'alternance des points de vue, d'une séquence à une autre, permet aux spectatrices et spectateurs de comprendre les raisons de chacun des personnages. La liste des principaux motifs de soaps établie par Modleski (la femme " maléfique », le grand sacrifice, la reconquête d'un/une amant(e) / époux(se), les unions vénales, la fille-mère, les tromperies autour de la paternité, l'opposition carrière - vie de famille, la femme alcoolique et parfois l'homme) ${ }^{15}$ est aussi la liste des problématiques principales des teen series. Dans plusieurs séries apparaît ainsi un personnage d'une rouerie et d'une méchanceté sans fond, généralement une femme, qui permet de cristalliser, comme il est fréquent dans les soap operas, les ambivalences des spectatrices. Dans la première saison de Gossip Girl, par exemple, les seuls personnages qui ne sont jamais

${ }^{14}$ Nous reviendrons sur ce point dans la suite de ce texte.

15 Tania Modleski, Loving With a Vengeance - Mass-Produced Fantasies for Women, op. cit., p. 78. 
rachetés par un aspect de leur conduite sont deux femmes, une ancienne amie proprement diabolique de Serena van der Woodsen (épisodes 1.15 à 1.18), et aussi la grand-mère de l'héroïne, prête à tous les mensonges et compromissions pour parvenir à ses fins et faire triompher ses idées rétrogrades (1.10).

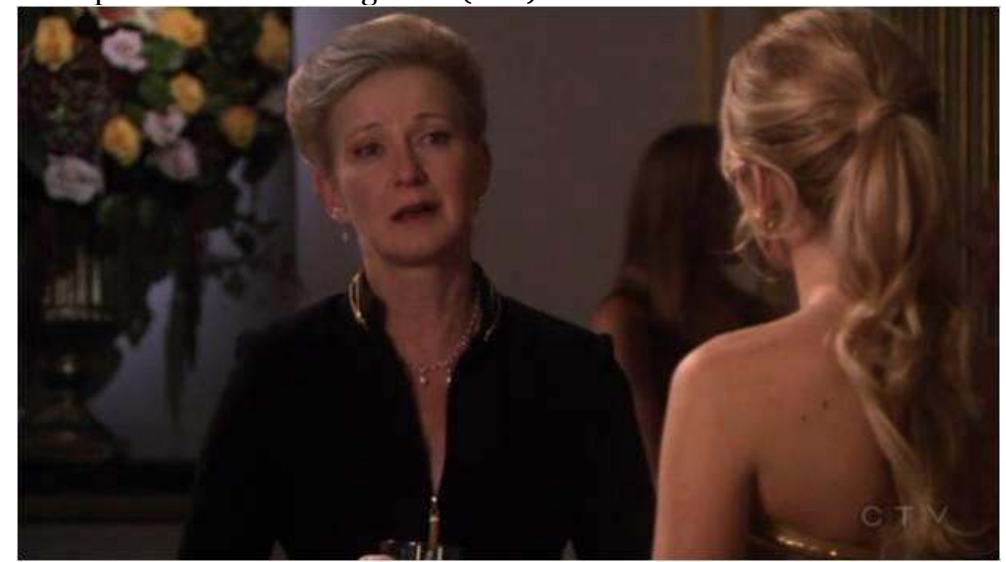

Gossip Girl

Dans les deux premières saisons de The Vampire Diaries, c'est tout d'abord le personnage de Damon Salvatore, le frère du héros, qui incarne un villain à la méchanceté abyssale. Mais Damon est vite racheté par son romantisme et son amour sans espoir pour Elena, et c'est Katherine, un personnage de vampire qui est le sosie de l'hérö̈ne, qui représente durablement le mal absolu. La même comédienne, Nina Dobrev, interprète Elena, une jeune fille mature et responsable ${ }^{16}$, et sa lointaine parente, la redoutable Katherine.

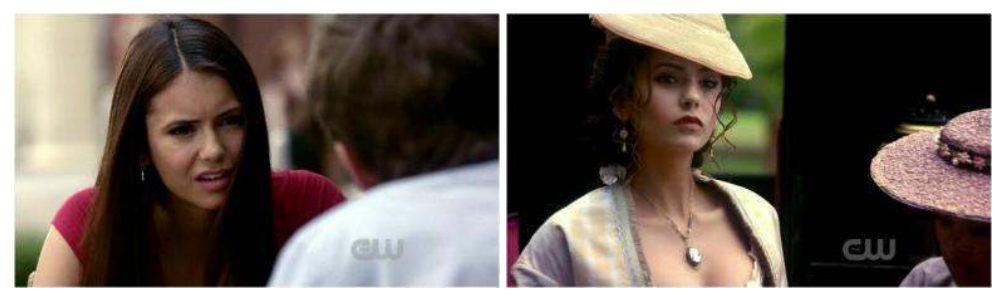

${ }^{16}$ A la suite du décès de ses parents, Elena protège de nombreux dangers son jeune frère Jeremy. Dans la saison 2 (cf épisode 2.9), quand elle apprend que sa famille et ses amis Tyler, Caroline et Bonnie sont menacés, le rôle maternel et sacrificiel d'Elena est accentué, elle est prête à mourir en martyr pour sauver ceux qu'elle aime. 
Le trouble est renforcé, dans plusieurs épisodes (cf. le début de l'épisode 1.20, en particulier), par un montage alterné et de nombreux raccords qui brisent une possible identification avec Elena en juxtaposant à cette figure positive l'image de son double maléfique.

\section{Identités multiples}

Le début de Veronica Mars éclaire aussi des enjeux qui étaient quelque peu occultés par une série comme Buffy, à savoir l'intersection dans les feuilletons des années 2000 des problématiques de genre, d'ethnicité, de classe sociale et de sexualité. Dans Listen Up, un ouvrage collectif dirigé par Barbara Findlen, une théoricienne de la troisième vague féministe ${ }^{17}$, les contributions explorent systématiquement les intersections entre ces identités multiples, et souvent contradictoires, qui sont celles des femmes (et aussi des hommes) d'aujourd'hui, tiraillées en particulier entre leurs obligations professionnelles et familiales, qui les empêchent de se définir prioritairement en termes d'identités sexuées. Or les teen series contemporaines proposent une vision neuve, dans le champ des séries, de cette question des identités multiples. Dans Buffy, la question se pose essentiellement par le biais du personnage de l'héroïne : jeune, blanche, hétérosexuelle, elle a le loisir de transiter et choisir entre ses identités de lycéenne, étudiante, tueuse de vampires, et femme active. Le mélange des genres est bien sûr une stratégie permettant de poser ces problématiques. Pourtant, quand sa position privilégiée est mise en péril (après la mort de sa mère, elle doit prendre un petit boulot dans un fast food), un retournement de situation (un chèque surprise de Giles, son mentor, et un travail de conseillère dans son ancien lycée) la tire miraculeusement de ce mauvais pas, et reconduit le présupposé post-féministe selon lequel tout est question de choix et de priorités individuelles - tout du moins pour les femmes de la classe moyenne, puisque la série n'aborde pas l'absence de choix qui est le lot des femmes moins favorisées, et opprimées par l'état de la société ${ }^{18}$.

Dans Veronica Mars, à l'inverse, la question du choix individuel se pose peu : dès le premier épisode, Veronica est présentée comme une jeune femme born on the wrong side of the tracks : parmi les enfants de stars ou de millionnaires du lycée de Neptune, elle est juste la fille du shérif local, et la série articule nettement la question identitaire autour du gender et des axes de la différence ethnique, sociale, ou encore sexuelle. Veronica est-elle qualifiée de freak dans les

\footnotetext{
${ }_{17}$ Lire par exemple Barbara Findlen (dir.), Listen up: Voices from the Next Feminist Generation, Seattle, Seal Press, 2001

${ }^{18}$ Nous reprenons ici l'analyse gender que fait Elana Levine de Buffy dans « Buffy and the "New Girl Order": Defining Feminism and Femininity », in Elana levine et Lisa Parks, op. cit., p. 168-190.
} 
premières minutes du pilote parce qu'elle est une "Mars woman », une femme qui endosse un rôle masculin, ou parce qu'elle est défavorisée socialement par rapport à ses camarades de lycée ? On retrouve cette articulation de l'identité sexuée et de l'identité de classe dans la plupart des teen series contemporaines. Dans Gilmore Girls comme dans Gossip Girl, un conflit difficilement surmontable est créé, chez les jeunes héroïnes, par une double appartenance - d'un côté un milieu familial middle class, et de l'autre un milieu scolaire huppé, puisqu'elles fréquentent une école privée où elles côtoient des enfants de familles très fortunées. Dans la première saison de Gossip Girl, cette double identité devient pour Jenny, l'une des héroïnes, l'objet d'une double oppression : elle est punie par son père, qui ne comprend pas les aspirations sociales de sa fille, et humiliée et sadisée par ses camarades de classe, notamment lors d'une fête d'anniversaire improvisée (1.14).
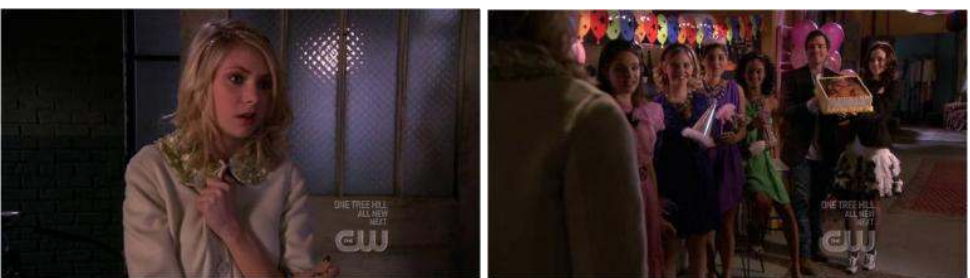

Dans ce type de série qui met en scène une situation d'oppression, la question post-féministe du choix individuel ne se pose plus, et la résolution de la tension est impossible. Pourtant, si Jenny est humiliée par ses camarades de classe richissimes, elle fait partie d'un milieu socio-culturel aisé, et cette appartenance à une upper middle-class désamorce quelque peu la problématique. Une série plus conservatrice comme The Vampire Diaries résout cette tension d'une manière réactionnaire dans sa première saison : l'une des héroïnes de la série, Vicki Donovan, est une jeune fille issue d'un milieu populaire, et qui ne trouve aucune issue à ses problèmes identitaires. 


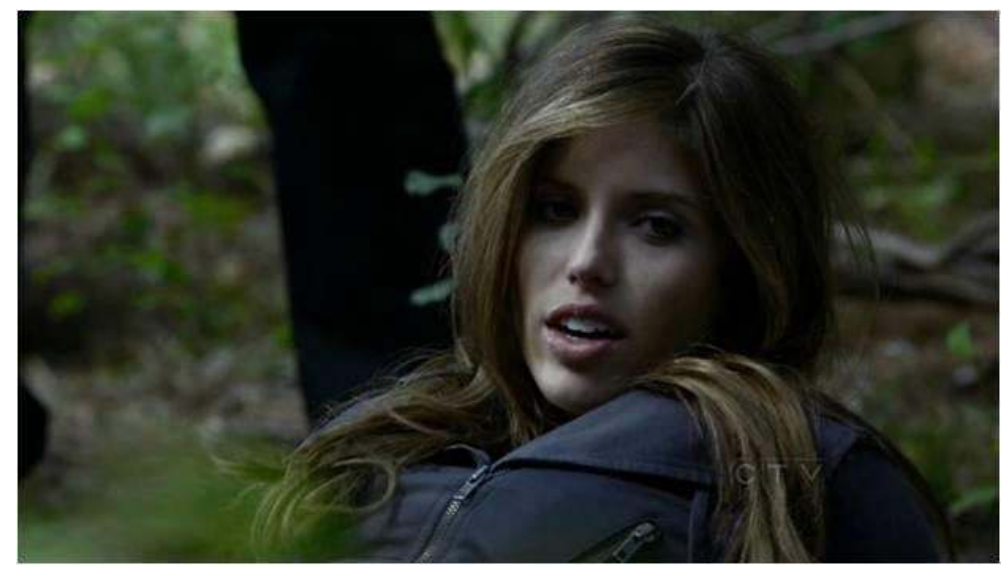

Son riche boyfriend, en particulier, ne s'oppose pas à ses parents qui voient d'un mauvais œil la relation de leur héritier avec cette fille peu recommandable. Il est alors symptomatique que Vicki soit une droguée, et surtout l'une des premières adolescentes à être mordue par un vampire, la seule enfin à être tuée car personne ne parvient à contrôler sa soif de sang (1.7), ce qui est une façon commode de résoudre les enjeux de classe... Il n'en demeure pas moins que les deux séries articulent identités sexuées et identité de classe, en montrant notamment que les frères des deux jeunes femmes peuvent franchir les barrières sociales avec une aisance qui est refusée à leur sœur, qui ne manque jamais de se révolter contre cet état de faits injuste.

Les séries abordent en revanche avec plus de timidité les enjeux ethniques : dans The Vampire Diaries, par exemple, une jeune fille noire, Bonnie, est l'amie de l'héroïne, Elena. Il s'avère vite que Bonnie est une lycéenne comme les autres, et aussi une sorcière aux grands pouvoirs: la série se contente alors de reprendre des stéréotypes associés aux gens de couleur. Dans la seconde saison de la série, Bonnie est même confrontée à une seconde famille de sorciers noirs, dans laquelle une jeune fille, Greta, met sa puissance au service des causes les moins recommandables. Bonnie, quant à elle, est une femme forte ${ }^{19}$, qui parvient à sauver son amie et aussi des hommes de situations bien périlleuses, notamment dans la seconde saison du feuilleton, mais son empowerment passe toujours par un rappel de ses pouvoirs de sorcière, et ce au prix d'approximations historiques ${ }^{20}$. Au

${ }^{19}$ La jeune fille est quelque peu instrumentalisée et figée dans son rôle de sorcière. Sa vie sentimentale, effleurée dans la seconde saison, passe au second plan, et la série préfère s'intéresser aux amours compliquées de jeunes filles blanches, Elena et Caroline.

${ }^{20}$ Cette lignée de sorcières est originaire de Salem, quand bien même les femmes qui furent jugées lors de cet épisode fameux de l'histoire des Etats-Unis n'étaient pas noires... 
début de la série, Bonnie est hantée par l'une de ses ancêtres, Emily, une sorcière aux redoutables pouvoirs. Or, en 1864, Emily était aussi l'esclave de Katherine, la jeune aristocrate qui est le sosie d'Elena.
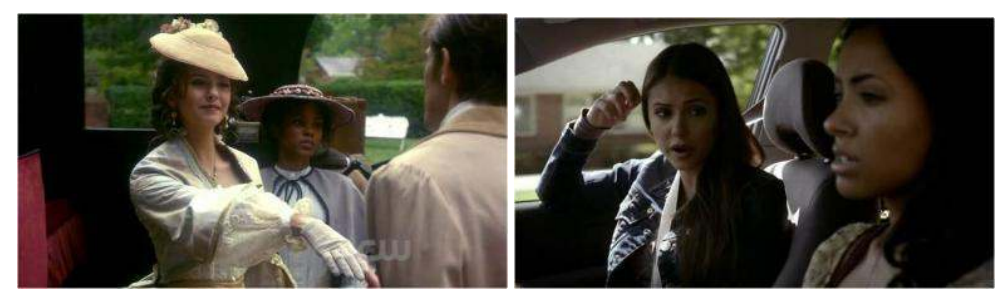

Si le parallèle entre le couple esclave / maitresse d'hier et celui formé par les deux amies aujourd'hui est explicite, il n'est pas l'occasion de souligner l'oppression que subit Bonnie, ou de contester la nature de la relation entre les deux jeunes femmes. L'une des qualités de la série comme d'ailleurs de toutes les séries de vampires - est de prendre en charge l'histoire de la nation américaine, et notamment l'épisode fondateur de la guerre de Sécession, mais elle se contente pourtant de reconduire une représentation convenue des rapports entre identité sexuée et identité ethnique: dans le dernier épisode de la seconde saison (2.22), une projection en plein air d'Autant en emporte le vent permet de juxtaposer des images du film, à l'arrière plan, et les dernières péripéties de la série, et donc d'établir des comparaisons entre hier et aujourd'hui : comme l'explique Caroline, «nous allons prendre exemple sur la vie de Scarlett. Nous avons traversé la guerre, je sais que vous avez vécu l'enfer, et ma mère sait que je suis une vampire. Donc c'est comme si Atlanta avait brûlé. Et pourtant, en dépit de tout cela, nous persévérons ${ }^{21}$ ». En matière de représentation du croisement entre identité sexuée et identité ethnique, rien n’a vraiment changé entre le film classique hollywoodien et la série de 2011.

Cette timidité ne doit cependant pas occulter l'audace dont font preuve les teen series contemporaines en matière de représentation des enjeux de sexe - domaine dans lequel elles s'inscrivent dans la filiation de Buffy contre les vampires. Elles témoignent d'une exploration précise des identités multiples des personnages, que l'on ne retrouve guère dans les autres genres sériels : alors que la plupart des séries dramatiques (Dexter, Mad Men, Breaking Bad, 24 heures chrono, etc.) qui ont renouvelé la fiction sérielle depuis une quinzaine d'années demeurent dans l'ensemble des

\footnotetext{
${ }^{21}$ "We are going to take a page from Scarlett. We made it through the war; I know you guys went through hell, and my mom knows I am a vampire, so basically it's like Atlanta has burnt. And yet, in spite of everything, we persevere ".
} 
mélodrames masculins qui perpétuent avec virulence la séparation sexuée des genres télévisuels ${ }^{22}$, les teen series explorent une voie fictionnelle alternative : en mettant en scène des personnages féminins complexes, elles continuent à s'adresser à un très large public. Mais elles laissent moins de place que les autres séries dramatiques à l'hérö̈sme masculin, et surtout renoncent généralement à valider une représentation stéréotypée des rapports de sexe.

22 Cf. Pierre-Olivier Toulza, «Le mode mélodramatique dans les séries télévisées américaines contemporaines ", in Dominique Nasta et Muriel Andrin (dir.), Le Mélodrame revu et corrigé, Peter Lang, à paraître en 2012. 